\section{Molecular Syndromology}

Mol Syndromol 2017;8:195-200

DOI: $10.1159 / 000476020$
Accepted: March 14, 2017

by M. Schmid

Published online: May 30, 2017

\title{
A Novel Pathogenic Variant in the MITF Gene Segregating with a Unique Spectrum of Ocular Findings in an Extended Iranian Waardenburg Syndrome Kindred
}

\author{
Nazanin Jalilian ${ }^{\mathrm{a}}$ Mohammad A. Tabatabaiefar, c Tayyeb Bahrami $^{\mathrm{d}}$ \\ Golaleh Karbasi $^{f}$ Mohammad H. Bahramian ${ }^{g}$ Abdolrahman Salimpoor ${ }^{g}$ \\ Mohammad R. Noori-Daloii ${ }^{\mathrm{C}}$ \\ a Department of Clinical Biochemistry, School of Medicine, Kermanshah University of Medical Sciences, Kermanshah, \\ ${ }^{b}$ Department of Genetics and Molecular Biology, School of Medicine, and 'Pediatric Inherited Diseases Research \\ Center, Research Institute for Primordial Prevention of Non-Communicable Disease, Isfahan University of Medical \\ Sciences, Isfahan, dGenetic Research Center, University of Social Welfare and Rehabilitation Sciences (USWR), \\ and ' Department of Medical Genetics, School of Medicine, Tehran University of Medical Sciences, Tehran, and \\ ${ }^{f}$ Kurdistan Welfare and Rehabilitation Organization, and ${ }^{9}$ School of Medicine, Kurdistan University of Medical \\ Sciences, Sanandaj, Iran
}

\section{Keywords}

Iran · MITF · Nystagmus - Optic disc hypoplasia .

Waardenburg syndrome type 2

\begin{abstract}
Waardenburg syndrome (WS) is a rare genetic disorder characterized by abnormal pigmentation of the hair, skin, and iris as well as sensorineural hearing loss. WS is subdivided into 4 major types (WS1-4), where WS2 is characterized by the absence of dystopia canthorum. This study was launched to investigate clinical and molecular characteristics of WS in an extended Iranian WS2 family. A comprehensive clinical investigation was performed. Peripheral blood samples were collected and genomic DNA was extracted. Affected members of the family were studied for possible mutations within the SOX10, MITF, and SNAI2 genes. Six WS2 individuals affected from a large Iranian WS2 kindred were enrolled. All affected members carried the novel substitution c.877C $>$ T at
\end{abstract}

\section{KARGER}

๑ 2017 S. Karger AG, Basel

E-Mail karger@karger.com

www.karger.com/msy exon 9 in the MITF gene, which resulted in p.Arg293* at the protein level. None of the healthy members and also of 50 ethnically matched controls had this variant. In addition, a spectrum of unique ocular findings, including nystagmus, chorioretinal degeneration, optic disc hypoplasia, astigmatism, and myopia, was segregated with the mutant allele in the pedigree. Our data provide insight into the genotypic and phenotypic spectrum of WS2 in an Iranian family and could further expand the spectrum of MITF mutations and have implications for genetic counseling on WS in Iran.

(c) 2017 S. Karger AG, Basel

Waardenburg syndrome (WS) is characterized by abnormal pigmentation of the hair, skin, and iris as well as varying degrees of sensorineural hearing impairment (HI). It is a rare genetic disorder with an autosomal dominant mode of inheritance. It can be classified into 4 major types (WS1-4), with 10 subtypes, based on the presence 
or absence of additional clinical features [Read and Newton, 1997; Pardono et al., 2003].

WS1 (OMIM 193500) and WS2 (OMIM 193510) are the most frequent types [Tamayo et al., 2008; Zaman et al., 2015]. WS2 can be distinguished from WS1 by the absence of dystopia canthorum, a lateral displacement of the inner canthi. The genes MITF, SOX10, and SNAI2 are considered to be the major loci involved [Tassabehji et al., 1994; Sánchez-Martín et al., 2002; Bondurand et al., 2007], which can explain the genetic basis of the syndrome in about $50 \%$ of the WS2 cases [Pingault et al., 2010].

MITF encodes a transcription factor that regulates differentiation and development of melanocytes and is also responsible for pigment cell-specific transcription of the melanogenesis enzyme genes [Bertolotto et al., 1998; Steingrímsson et al., 2004; Hou and Pavan, 2008]. Heterozygous mutations in this gene cause WS2 as well as Tietz syndrome (OMIM 103500) [Smith et al., 2000]. Tietz syndrome is characterized by severe $\mathrm{HI}$ and generalized hypopigmentation of the eyes, hair and skin, rather than the patchy depigmentation observed in WS [Tietz, 1963].

MITF is a basic helix-loop-helix transcription factor which plays a key role in melanocyte development [Steingrímsson et al., 2004]. The MITF gene has 9 alternative promoters and a complex pattern of alternative splicing, which leads to different isoforms differing at their $\mathrm{N}$ termini (17 transcripts and 14 proteins) [Hershey and Fisher, 2005]. Isoform M (for melanocyte) encodes a 419 amino acid protein that is extensively expressed in melanocytes [Tassabehji et al., 1994]. Besides WS2, mutations and aberrant expression of MITF have been observed in melanoma [Steingrímsson et al., 2004].

The WS subtype associated with MITF mutations is known as WS2A. To date, nearly 50 variants have been detected in this gene (http://www.hgmd.cf.ac.uk/ac/all. php); however, the Iranian population has not been subject of investigation. The Iranian population has a unique heterogeneous structure and extended pedigrees available; hence, it can be regarded as a suitable subject for mutational analysis, gene discovery, and genotype-phenotype correlation studies. Following our previous studies on the genetic basis of WS in Iranian population [Jalilian et al., 2015a, b], herein, we report an extended Iranian family clinically suspected with WS2. Our investigations resulted in identification of a novel pathogenic variant in MITF segregating with additional ocular findings which were not previously reported in association with MITF pathogenic variants.

\section{Patients and Methods}

The family was referred to our laboratory by a genetic counseling center affiliated to the Welfare and Rehabilitation Organization, Sanandaj, Kurdistan province, Iran. A comprehensive clinical history was taken, and audiological, ophthalmological, and dermatological examinations were performed. Peripheral blood samples were collected in $0.5 \mathrm{~mm}$ EDTA-containing tubes.

\section{Molecular Analysis}

Genomic DNA was extracted from peripheral venous blood using AccuPrep ${ }^{\circledR}$ Genomic DNA Extraction Kit (Bioneer, South Korea), and 50 ng were used as a template in PCR reactions. Affected members of the family were studied for possible mutations in the SOX10, MITF, and SNAI2 genes. The primers to amplify all exons and exon-intron boundaries of the studied genes as well as PCR conditions are available upon request. Subsequently, all PCR products were sequenced bidirectionally using ABI3130 automated sequencer (Macrogen, South Korea) and analyzed using Chromas version 2 .

\section{Validation of the Variants}

We used the following approach in order to validate any variant found in the study; this approach was based on the American College of Medical Genetics and Genomics guidelines for the interpretation of sequence variants [Richards et al., 2015]: (1) segregation analysis of the whole family: all apparently normal and affected members were screened for the possible mutation using direct sequencing; (2) searching through the population: 50 healthy controls of the same ethnicity were screened along with the patients using sequencing; (3) searching population and disease-specific databases: public databases were investigated including Ensemble. org, dbSNP (https://www.ncbi.nlm.nih.gov/snp/), ClinVar (http:// www.ncbi.nlm.nih.gov/clinvar/), Exome Variant Server (http:// evs.gs.washington.edu/EVS/), and 1000 Genome databases (http:// browser.1000genomes.org/) and (4) in silico predictive analyses: sequence variant numbering was based on the transcript ENST00000394351 for MITF (NM_000248). The variant was named according to the guidelines of the Human Genome Variation Society (http://www.hgvs.org/). The possible pathogenic effect of the novel variant was checked by the Mutation Taster. The tertiary structure of normal and mutant protein was predicted using the I-TASSER server [Zhang, 2008; Roy et al., 2010; Yang et al., 2015].

\section{Results}

\section{Clinical Findings}

The pedigree IR-WS-03 was ascertained through premarital genetic counseling, as couple III.6 and III.7 (first cousins) were concerned about the risk of $\mathrm{HI}$ in their future children. The couple had normal hearing. However, HI in association with pigmentary disturbances of hair and eyes segregates through the pedigree with an apparent dominant mode of inheritance. WS2 diagnosis was suspected based on clinical manifestations according to the interna-
196

Mol Syndromol 2017;8:195-200 DOI: $10.1159 / 000476020$
Jalilian/Tabatabaiefar/Bahrami/Karbasi/ Bahramian/Salimpoor/Noori-Daloii 


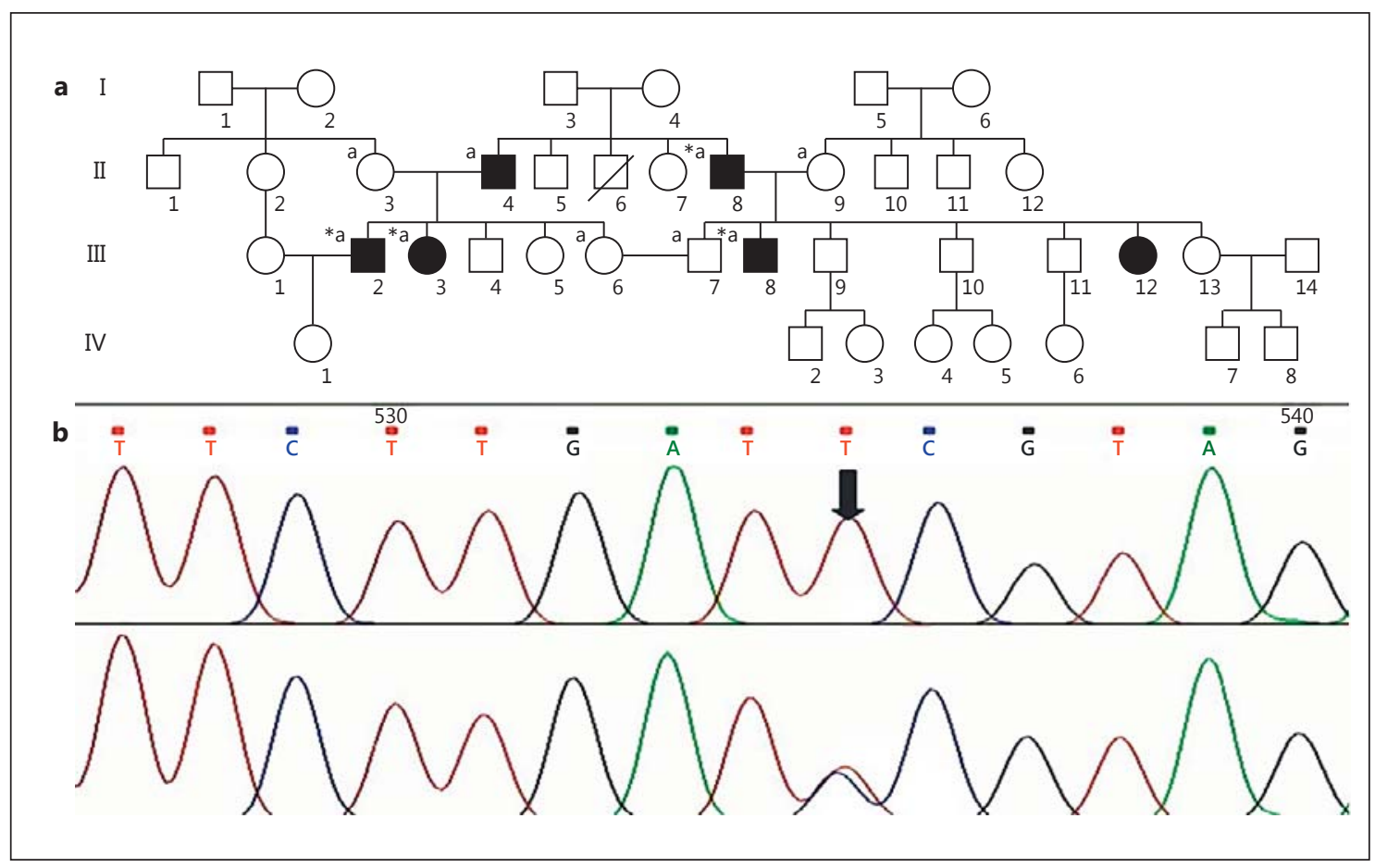

Fig. 1. a Pedigree of the family IR-WS-03. Filled squares/circles denote the affected members with clinical symptoms. *, individuals who were clinically investigated; a, individuals who have undergone DNA analysis. b Electropherograms of the normal and mutated MITF gene.

tional criteria proposed by the Waardenburg Consortium [Farrer et al., 1992]. Further clinical investigations revealed a WS2 pedigree with 6 affected members in 2 consanguineous loops (Fig. 1). Detailed clinical descriptions of affected members are described below (II.4 refused to participate in clinical investigations; however, sensorineural $\mathrm{HI}$ and hypoplastic blue eyes were present by hearsay).

II.8 was a 77-year-old man suffering from severe to profound $\mathrm{HI}$ and pigmentary disturbances of the hair as well as heterochromia iridis with a left hypoplastic blue eye. The right eye was totally blind due to advanced glaucoma and had no light perception. Ocular exams also revealed counting fingers at $4 \mathrm{~m}(4 \mathrm{~m} \mathrm{CF})$, and the fundoscopic exam depicted evidence of severe myopia at the left fundus.

III. 2 was a 35 -year-old man with moderate sensorineural HI, corrected with hearing aids. He had a white forelock and his hair started graying at the age of 20. III.2 had partial heterochromia with a visual acuity of $10 / 10$ in both eyes.

III. 3 was a 33 -year-old female affected with severe to profound HI, with a white forelock emerging at 10. She presented with segmental heterochromia iridis with no

Novel Pathogenic Variant in MITF cataract, and her visual acuity was $6 / 10$ for the right eye and 9/10 for the left eye. The optic disc also showed temporal atrophy in both eyes and fundoscopy revealed evidence of chorioretinal degeneration.

III. 8 was 30 years old. He presented with profound HI, white forelock, and early graying, with a hypoplastic left iris. He showed nystagmus in both eyes with visual acuity of $1 / 10$ for the right and 3/10 for the left eye. He also had small optic discs, leading to decreased visual acuity and nystagmus. Fundoscopy results of case III.8 are depicted in Figure 2.

III.12 was a 23-year-old female patient with a white forelock but with normal hearing and visual acuity (10/10 for both eyes). The cornea was vivid, and a cataract was not present. However, fundoscopy revealed levels of choriretinal degeneration which did not affect vision.

\section{Molecular Findings}

All affected members carried the novel substitution c. $877 \mathrm{C}>\mathrm{T}$ at exon 9 in MITF, while none of the healthy members and of the 50 ethnically matched controls had this variant. The c. $877 \mathrm{C}>\mathrm{T}$ transversion results in p.Arg293* at the protein level, located at the intervening 
Fig. 2. The left fundus and the right fundus of case III.8. The areas of hypopigmentation and disc hypoplasia are evident. OS, oculus sinister; OD, oculus dexter.
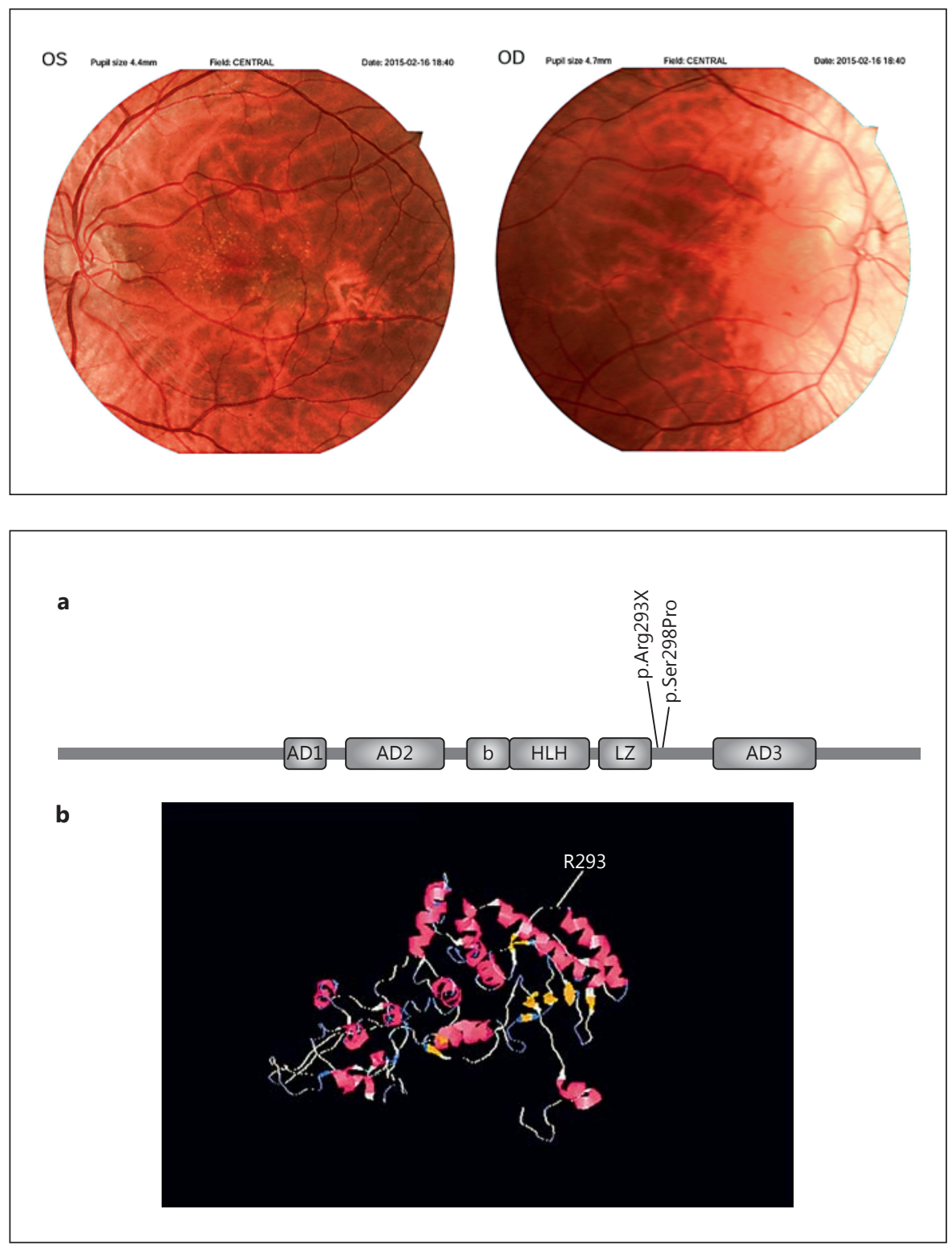

Fig. 3. a MITF structure and localization of the variant p.Arg293X observed in this study. b Localization of Arg293 in the predicted 3D structure of the protein.

sequence of the leucin zipper and transactivation domain, 5 amino acids downstream of the leucine zipper (Fig. 3).

In order to further delineate the pathogenicity of c. $877 \mathrm{C}>\mathrm{T}$, a comprehensive computational analysis was performed. The nonsense mutation occurred in a conserved region and was predicted as pathogenic by Mutation Taster.

Based on the American College of Medical Genetics and Genomics and the College of American Pathologists guidelines, this variant was considered to be pathogenic [Richards et al., 2015]: (1) the nucleotide substitution generates a null variant, which by itself is very strong evidence in favor of pathogenicity (PVS1); (2) the inheri- tance pattern of this variant is autosomal dominant (PP1), and (3) this variant was found neither in 50 healthy ethnically matched controls nor in population databases including exome sequence variant server, databases SNP, and databases variant (PM2).

\section{Discussion}

In this study, we introduce a novel pathogenic variant in the MITF gene. Most of the MITF mutations identified so far are distributed in exons 7 and 8 . These 2 exons encode the basic domain, HLH, and parts of the leucin zip- 
per domain. The mutation p.R293X, found in this study, is located in exon 9 at the intervening sequence of the leucine zipper and the $3 \mathrm{rd}$ transactivation domain of the protein AD3 (Fig. 3), where only 1 mutation (p.S298P) has been reported so far [Takeda et al., 2000].

Several speculations concerning the effect of the p.R293X mutation on functionality can be made. First, this premature stop codon abolishes a transactivation domain of the protein $\mathrm{AD} 3$, hence affecting the transactivating capacity of MITF. Second, Ser298 seems to be a critical position in MITF as in vitro studies have shown that phosphorylated Ser298 enhances binding of MITF to DNA [Takeda et al., 2000]; therefore, as a result of the truncated protein, phosphorylation of MITF by GSK3beta is abolished, and MITF function is impaired. Third, this premature stop codon abolishes 127 amino acids (more than $10 \%$ of the protein length); therefore, nonsense-mediated decay could be considered a possible underlying mechanism. Functional studies are required to clarify the exact effect of the variant on the protein function.

WS2 shows considerable clinical and genetic heterogeneity. HI (77\%) followed by heterochromia iridis (47\%) are the most frequent features [Liu et al., 1995], which are also the most penetrant findings in our studied family (4 out of 5 affected members). The pedigree also demonstrates obvious variable expression among affected members. Notably, varying degrees of HI were prominent, ranging from normal hearing to moderately impaired and profound hearing loss. Heterochromia was also found as both segmental and complete. This clinical heterogeneity within a family is not new to WS2 and has been observed in different reports of different ethnicities; for example, a Turkish family with 2 affected sons has been reported where the younger brother had strabismus, profound HI, and abnormal fundus pigmentation with no heterochromia. However, his brother had complete heterochromia with a hypoplastic blue left iris and sensorineural HI with moderate impairment on the right and profound hearing loss on the left ear. A causative variant was not found in the family [Müllner-Eidenböck et al., 2001].

Here, we made some interesting observations on the clinical findings of WS2 as a unique spectrum of ocular findings, including nystagmus, chorioretinal degeneration, optic disc hypoplasia, astigmatism, and myopia segregating with the mutant allele through the pedigree. To the best of our knowledge, these findings were rarely, if ever, reported in association with MITF mutations, mostly as an isolated ocular finding, and to our knowledge, this

Novel Pathogenic Variant in MITF is the first report of such a spectrum. Nystagmus has been also reported with SOX10 mutations [Pingault et al., 2000]. Strabismus and amblyopia have been previously reported to be associated with MITF mutations [Léger et al., 2012].

Few genotype-phenotype correlations have been speculated for MITF mutations. Léger et al. [2012] found a high rate $(40 \%)$ of ocular abnormalities in their studied population with MITF basic domain mutations, leading to the possibility that they could be more frequently or specifically associated with mutations of this domain. However, it could not be applied to our study since the variant we found here, p.R293X, is not located within the basic domain. Additional similar observations and also functional studies are required in order to decide whether these ocular findings are truly associated with MITF mutations.

In this study, we performed a comprehensive genetic and clinical study on an Iranian WS2 family which led to the identification of a novel pathogenic variant in MITF. This novel variant could contribute to the expansion of the MITF mutational spectrum. We also demonstrated several ocular features that were unique to MITF mutations and could further expand the phenotypic spectrum of MITF mutations and provide better insight into diagnosis and genetic counseling on WS.

\section{Acknowledgment}

We would like to express our gratitude to all participants in this study. This research was supported by the Tehran University of Medical Sciences (grant 21446).

\section{Statement of Ethics}

This work was approved by the Ethics Committee of the Tehran University of Medical Sciences. Informed consent was obtained prior to the investigation.

\section{Disclosure Statement}

The authors declare no conflicts of interest. 


\section{References}

Bertolotto C, Buscà R, Abbe $\mathrm{P}$, Bille K, Aberdam $\mathrm{E}$, et al: Different cis-acting elements are involved in the regulation of TRP1 and TRP2 promoter activities by cyclic AMP: pivotal role of $\mathrm{M}$ boxes (GTCATGTGCT) and of microphthalmia. Mol Cell Biol 18:694-702 (1998).

Bondurand N, Dastot-Le Moal F, Stanchina L, Collot N, Baral V, et al: Deletions at the SOX10 gene locus cause Waardenburg syndrome types 2 and 4. Am J Hum Genet 81: 1169-1185 (2007).

Farrer LA, Grundfast KM, Amos J, Arnos KS, Asher JH Jr, et al: Waardenburg syndrome (WS) type I is caused by defects at multiple loci, one of which is near ALPP on chromosome 2: first report of the WS consortium. Am J Hum Genet 50:902-913 (1992).

Hershey CL, Fisher DE: Genomic analysis of the microphthalmia locus and identification of the MITF-J/Mitf-J isoform. Gene 347:73-82 (2005).

Hou L, Pavan WJ: Transcriptional and signaling regulation in neural crest stem cell-derived melanocyte development: do all roads lead to Mitf? Cell Res 18:1163-1176 (2008).

Jalilian N, Tabatabaiefar MA, Farhadi M, Bahrami T, Emamdjomeh H, Noori-Daloii MR: Molecular and clinical characterization of Waardenburg syndrome type I in an Iranian cohort with 2 novel PAX3 mutations. Gene 574:302-307 (2015a).

Jalilian N, Tabatabaiefar MA, Farhadi M, Bahrami T, Noori-Daloii MR: A novel mutation in the PAX3 gene causes Waardenburg syndrome type I in an Iranian family. Int J Pediatr Otorhinolaryngol 79:1736-1740 (2015b).
Léger S, Balguerie X, Goldenberg A, Drouin-Garraud $V$, Cabot $A$, et al: Novel and recurrent non-truncating mutations of the MITF basic domain: genotypic and phenotypic variations in Waardenburg and Tietz syndromes. Eur J Hum Genet 20:584-587 (2012).

Liu XZ, Newton VE, Read AP: Waardenburg syndrome type II: phenotypic findings and diagnostic criteria. Am J Med Genet 55:95-100 (1995).

Müllner-Eidenböck A, Moser E, Frisch H, Read AP: Waardenburg syndrome type 2 in a Turkish family: implications for the importance of the pattern of fundus pigmentation. $\mathrm{Br} \mathrm{J}$ Ophthalmol 85:1384-1386 (2001).

Pardono E, van Bever Y, van den Ende J, Havrenne PC, Iughetti P, et al: Waardenburg syndrome: clinical differentiation between types I and II. Am J Med Genet A 117A:223-235 (2003).

Pingault V, Guiochon-Mantel A, Bondurand N, Faure C, Lacroix C, et al: Peripheral neuropathy with hypomyelination, chronic intestinal pseudo-obstruction and deafness: a developmental "neural crest syndrome" related to a SOX10 mutation. Ann Neurol 48:671-676 (2000).

Pingault V, Ente D, Dastot-Le Moal F, Goossens M, Marlin S, Bondurand N: Review and update of mutations causing Waardenburg syndrome. Hum Mutat 31:391-406 (2010).

Read AP, Newton VE: Waardenburg syndrome. J Med Genet 34:656-665 (1997).

Richards S, Aziz N, Bale S, Bick D, Das S, et al: Standards and guidelines for the interpretation of sequence variants: a joint consensus recommendation of the American College of Medical Genetics and Genomics and the Association for Molecular Pathology. Genet Med 17:405-424 (2015).

Roy A, Kucukural A, Zhang Y, I-TASSER: a unified platform for automated protein structure and function prediction. Nat Protoc 5:725738 (2010).
Sánchez-Martín M, Rodríguez-García A, PérezLosada J, Sagrera A, Read AP, Sánchez-García I: SLUG (SNAI2) deletions in patients with Waardenburg disease. Hum Mol Genet 11: 3231-3236 (2002).

Smith SD, Kelley PM, Kenyon JB, Hoover D: Tietz syndrome (hypopigmentation/deafness) caused by mutation of MITF. J Med Genet 37: 446-448 (2000).

Steingrímsson E, Copeland NG, Jenkins NA: Melanocytes and the microphthalmia transcription factor network. Annu Rev Genet 38:365411 (2004).

Takeda K, Takemoto C, Kobayashi I, Watanabe A, Nobukuni Y, et al: Ser298 of MITF, a mutation site in Waardenburg syndrome type 2, is a phosphorylation site with functional significance. Hum Mol Genet 9:125-132 (2000).

Tamayo ML, Gelvez N, Rodriguez M, Florez S, Varon C, et al: Screening program for Waardenburg syndrome in Colombia: clinical definition and phenotypic variability. Am J Med Genet A 146A:1026-1031 (2008).

Tassabehji M, Newton VE, Read AP: Waardenburg syndrome type 2 caused by mutations in the human microphthalmia (MITF) gene. Nat Genet 8:251-255 (1994).

Tietz W: A syndrome of deaf-mutism associated with albinism showing dominant autosomal inheritance. Am J Hum Genet 15:259-264 (1963).

Yang J, Yan R, Roy A, Xu D, Poisson J, Zhang Y: The I-TASSER Suite: protein structure and function prediction. Nat Methods 12:7-8 (2015).

Zaman A, Capper R, Baddoo W: Waardenburg syndrome: more common than you think! Clin Otolaryngol 40:44-48 (2015).

Zhang Y: I-TASSER server for protein 3D structure prediction. BMC Bioinformatics 9:40 (2008). 